\title{
Rapid tests: preliminar identification from blood culture
}

\author{
Gobbato Elisa, Kroumova Vesselina, Macaluso Paola, Tamburelli Sabrina, Mucedola Luca, \\ Fortina Giacomo \\ A.O.U. “Maggiore della Carità", S.C.D.O. Laboratorio di Microbiologia e Virologia, Novara
}

Key words: Rapid test, Bacterial identification, Blood culture

Test rapidi: identificazione preliminare da emocolture

\section{SUMMARY}

In the period I April to 30 June $20 \mathrm{II}$, we compared the rapid test identification of 250 positive blood cultures with the identification by MALDI-TOF mass spectrometer (Bruker).

After Gram staining and an important step in HB\&L liquid culture (Alifax), rapid tests were performed according to the morphological characteristics of the sample.

Pneumococcus urinary antigen test and tube coagulase test showed a concordance of $100 \%$, the concordance rates for Pyr test and oxidase test were $91 \%$ and $89 \%$ respectively.

Overall, the percentage of agreement of the rapid tests on 250 samples was found to be of $99.2 \%$.

The data obtained show that, even with their increased bacterial concentration due to the enrichment step in HB\&L liquid culture, it is possible to obtain preliminary identification directly from positive blood culture with simple rapid tests.

\section{INTRODUZIONE}

La diagnostica batteriologica è sempre più attenta alla sua ricaduta clinica e quindi al suo impatto nella cura del singolo paziente. Per questo motivo, e anche grazie a nuove tecnologie come la biologia molecolare, la spettrometria di massa ed altre ancora, il microbiologo clinico tende ad offrire risposte sempre più rapide al clinico.

Purtroppo l'utilizzo di queste nuove tecnologie spesso non risulta agevole, attualmente per tutti i laboratori sia per il loro costo, sia per l'impegno di personale che spesso richiedono (2).

Abbiamo perciò pensato di valutare se attraverso l'utilizzo di metodiche tradizionali come i test rapidi, era possibile ottenere informazioni utili senza importanti investimenti economici non sempre disponibili per tutti i laboratori.

\section{MATERIALI E METODI}

Nel periodo 1 Aprile - 30 Giugno 2011 sono state analizzate 250 emocolture positive (BACTECBD) comparando la loro identificazione, attraverso test rapidi, con quella ottenuta mediante lo spettrometro di massa MALDI-TOF (Bruker).

Non sono state prese in considerazione, in questo studio, le positività per miceti.

Come da metodica da noi già precedentemente pubblicata (2), dopo colorazione di Gram le emocolture sono state inoculate in vials per coltura liquida sullo strumento HB\&L (Alifax) a $37^{\circ} \mathrm{C}$.
Al raggiungimento di una torbidità di circa 0.5 $\mathrm{McF}$, sono stati allestiti i test rapidi in funzione delle caratteristiche morfologiche dei batteri presenti (3).

In caso di microrganismi Gram positivi disposti a grappolo è stato allestito il test della Coagulasi in Tubo. In un'aliquota di $500 \mu \mathrm{L}$ di plasma di coniglio (BD) sono stati introdotti $50 \mu \mathrm{L}$ di campione e la provetta è stata successivamente posta a bagnomaria a $37^{\circ} \mathrm{C}$. La provetta è rimasta in osservazione per 4 ore. In caso di positività si verificava una gelificazione del campione (4).

Per i microrganismi Gram positivi disposti in catenelle è stato allestito il Pyr Test. È stato prelevato circa $1 \mathrm{~mL}$ di campione sempre alla concentrazione di $0.5 \mathrm{McF}$ e centrifugato a $13000 \mathrm{rpm}$ per 3 minuti. Il pellet così ottenuto è stato stemperato su una Pyr card (BD) precedentemente idratata. In caso di positività la card virava al colore rosa, viceversa, in assenza di viraggio, il Pyr test era da considerarsi negativo (6).

Per il test dell'Antigene urinario dello Streptococcus pneumoniae il campione utilizzato per il test precedente è stato risospeso. Un tampone imbevuto della sospensione in esame è stato successivamente inserito nella card immunocromatografica (Binax). La comparsa dopo 15 minuti di una banda oltre a quella del controllo, era segno di positività per il campione in esame (7).

Nel caso di microrganismi Gram negativi è stato

\section{Corresponding author: Elisa Gobbato}

A.O.U. “Maggiore della Carità”, Laboratorio di Microbiologia e Virologia,

C.so Mazzini, 18 - 28I00 Novara - Tel.: 032I 3733595

E-mail: gobbatoelisa@libero.it 
allestito il test dell'Ossidasi. È stato prelevato circa $1 \mathrm{~mL}$ di campione a $0.5 \mathrm{McF}$ e centrifugato a $13000 \mathrm{rpm}$ per 3 minuti. Il pellet così ottenuto è stato stemperato su una Oxidase card (BD). Se positivo la card virava verso un colore viola intenso (5). L'identificazione delle emocolture positive è stata effettuata mediante spettrometro di massa MALDI-TOF attraverso la metodica già da noi descritta precedentemente $(1,2)$.

\section{RISULTATI}

Le 250 emocolture positive analizzate hanno mostrato alla colorazione di Gram la presenza di 91 campioni Gram positivi successivamente identificati mediante spettrometro di massa MALDITOF (Bruker) e da 159 campioni Gram positivi, anch'essi confermati dall'identificazione mediante MALDI-TOF (Bruker) (Tabella 1).

Il test dell'Ossidasi eseguito sui 91 campioni Gram negativi ha mostrato una positività in 9 campioni, a fronte di solo 8 campioni (8 ceppi di Pseudomonas aeruginosa) con caratteristiche fenotipiche di positività al test dell'Ossidasi (Tabella 2).

I 159 campioni Gram positivi erano ulteriormente divisi fra campioni che mostravano la presenza di cocchi Gram positivi a grappolo e campioni con disposizione dei cocchi a catenella.

Questi ultimi 24 campioni sono stati sottoposti all'analisi del Pyr test.

Per 10 di questi 24 campioni è stata riscontrata una positività al Pyr test e quindi sono stati seminati su Agar sangue per evidenziare il tipo di emolisi e differenziare gli Streptococchi $\beta$-emolitici da Enterococcus spp. I rimanenti 14 campioni sono stati sottoposti alla ricerca dell'Antigene di Streptococcus pneumoniae con esito positivo in 5 casi, rilevando in questo modo la presenza di 5 Streptococcus pneumoniae. I rimanenti 9 campioni sono stati quindi ritenuti Streptococchi non $\beta$-emolitici (Tabelle 3 e 4).

L'identificazione fenotipica con metodicche tradizionali ha evidenziato un ceppo di Enterococcus faecalis che nella nostra casistica era risultato pyr negativo e identificato quindi di default come Streptococcus spp.
Dei 135 cocchi a grappolo, 28 sono risultati coagulasi positivi. Tutti questi ceppi sono stati identificati da MALDI-TOF come $S$. aureus, mentre i restanti 107 sono risultati negativi al test della coagulasi e tutti identificati come SCN (Tabella 5).

In definitiva, dei 250 campioni esaminati, solo in due casi i test rapidi non sono stati in grado di indicare una corretta identificazione batterica, inoltre, la sensibilità dei test rapidi è risultata essere conseguentemente del 100\% per Coagulasi, Ossidasi e Antigene urinario dello Streptococcus pneumoniae, mentre si è rivelata del $91 \%$ per il Pyr test.

La specificità, invece, si è rivelata essere del 100\% per Coagulasi, Pyr test e Antigene urinario per lo Streptococcus pneumoniae mentre per l'Ossidasi la specificità è risultata essere del $98.8 \%$.

Nel complesso la percentuale di concordanza dei test rapidi sui 250 campioni esaminati è risultata essere del $99.2 \%$.

\begin{tabular}{|c|c|}
\hline MICRORGANISMO & $\mathbf{N}^{\circ}$ \\
\hline Enterobatteri & 80 \\
\hline P. aeruginosa & 9 \\
\hline Altri Non Fermentanti & 1 \\
\hline S. aureus & 28 \\
\hline $\mathrm{SCN}$ & 107 \\
\hline Streptococcus spp. & 8 \\
\hline S. pneumoniae & 5 \\
\hline Enterococcus spp. & 11 \\
\hline Altri & $\mathrm{I}$ \\
\hline
\end{tabular}

\section{CONCLUSIONI}

I risultati così ottenuti hanno mostrato che, probabilmente anche grazie ad un veloce passaggio in brodo HB\&L (Alifax) che consente un arricchimento della popolazione batterica presente nel campione da esaminare, è possibile, senza avere a disposizione nuove e costose tecnologie, ottenere utili informazioni già pochissimo tempo dopo la positivizzazione dell'emocoltura senza dover attendere i tradizionali due giorni per un'identificazione tradizionale che, ovviamente, dovrà essere effettuata anche dopo tali risultati preliminari, ma che servirà solo per confermare o dare maggior completezza alla risposta stessa.

Tabella 2. Dati relativi al test dell'Ossidasi su microrganismi Gram negativi.

\begin{tabular}{lccccc}
\hline & N. & OSS + & OSS- & $\begin{array}{c}\text { Ceppi identificati } \\
\text { OSSIDASI + }\end{array}$ & $\begin{array}{c}\text { Ceppi identificati } \\
\text { OSSIDASI - }\end{array}$ \\
\hline GRAM NEGATIVI & 91 & 9 & 82 & 8 & 83 \\
\hline
\end{tabular}

Tabella 3. Dati relativi al Pyr test su microrganismi Gram positivi disposti in catenella.

\begin{tabular}{lccccc}
\hline & N. & PYR & PYR- & $\begin{array}{c}\text { Ceppi identificati } \\
\text { Enterococcus spp. }\end{array}$ & $\begin{array}{c}\text { Ceppi identificati } \\
\text { Str. non B-emolitici }\end{array}$ \\
\hline $\begin{array}{l}\text { GRAM POSITIVI } \\
\text { IN CATENELLA }\end{array}$ & 24 & 10 & 14 & 11 & 13 \\
\hline
\end{tabular}


Tabella 4. Dati relativi al test dell'Antigene urinario dello S. pneumoniae su microrganismi Gram positivi in catenella

\begin{tabular}{cccccc}
\hline & N. & Ag Urinario & Ag Urinario & Ceppi identificati & Ceppi identificati \\
\hline & & S. pneumoniae + & S. pneumoniae & S.pneumoniae & Streptococcus spp. \\
\hline Streptococcus spp. & 13 & 5 & 8 & 5 & 8
\end{tabular}

Tabella 5. Dati relativi al test della Coagulasi su microrganismi Gram positivi disposti a grappolo.

N. COAGULASI + COAGULASI - Ceppi identificati Ceppi identificati

\begin{tabular}{lllccc} 
& & & & S. aureus & SCN \\
\hline $\begin{array}{l}\text { GRAM POSITIVI } \\
\text { A GRAPPOLO }\end{array}$ & 135 & 28 & 107 & 28 & 107 \\
\hline
\end{tabular}

Una tale rapidità di informazione trasferita al clinico accoppiata ad una precisa ed aggiornata situazione epidemiologica delle resistenze presenti permetterà l'impostazione, in anticipo, di una terapia più adeguata determinando in tal modo un indubbio vantaggio per il paziente.

\section{BIBLIOGRAFIA}

1. Bizzini A, Durussel C, Bille J, Greub G, Prod'hom G. Performance of Matrix-Assisted Laser Desorption Ionization-Time of Flight Mass Spectrometry for identification of bacterial strains routinely isolated in a clinical microbiology laboratory. $J$ Clin Microb. 2010; 48(5): 1549-54.

2. Kroumova V, Gobbato E, Basso E, Mucedola L, Giani T, Fortina G. Direct identification of bacteria in blood culture by matrix-assisted laser desorption/ionization time-of-flight mass spectrometry: a new methodological approach. Rapid Commun Mass Spectrom 2011;
25(15): 2247-9.

3. Kroumova V, Gobbato E, Macaluso $P$, et al. Preliminary indication for antibiotic susceptibility tests in less than six hours in positive blood cultures. Microbiologia Medica. 2010; 25(1): 24-6.

4. McDonald CL, Chapin K. Rapid identification of Staphylococcus aureus from blood culture bottles by a classic 2-hour Tube Coagulase Test. J Clin Microbiol. 1995; 33(1): 50-2.

5. NHS; National Standard Method. Oxidase Test BSOP TP 26 Issued by Standard Unit, Department for Evaluations, Standards and Training Centre for Infections. 2010; Issue No 2.1.

6. Murray PR, et al. Manual of Clinical Microbiology, $8^{\text {th }}$ ed.merican Society for Microbiology, Washington, D.C. $2003 ; 360$.

7. Smith MD, Sheppard CL, Hogan A, et al. Diagnosis of Streptococcus pneumoniae infections in adults with bacteremia and Community-Acquired Pneumonia: clinical comparison of pneumococcal PCR and urinary antigen detection. J Clin Microbiol. 2009; 47(4): 1046-9. 